Case Report

\title{
Necrotizing ANCA-Positive Glomerulonephritis Secondary to Culture-Negative Endocarditis
}

\author{
Sophie Van Haare Heijmeijer, ${ }^{1}$ Dunja Wilmes, ${ }^{2}$ Selda Aydin, ${ }^{3}$ \\ Caroline Clerckx, ${ }^{1}$ and Laura Labriola ${ }^{4}$ \\ ${ }^{1}$ Department of Nephrology, Clinique Saint-Pierre, Ottignies, Belgium \\ ${ }^{2}$ Department of Internal Medicine, Cliniques universitaires Saint-Luc, Université catholique de Louvain, Brussels, Belgium \\ ${ }^{3}$ Department of Pathology, Cliniques universitaires Saint-Luc, Université catholique de Louvain, Brussels, Belgium \\ ${ }^{4}$ Department of Nephrology, Cliniques universitaires Saint-Luc, Université catholique de Louvain, Brussels, Belgium
}

Correspondence should be addressed to Laura Labriola; laura.labriola@uclouvain.be

Received 26 October 2015; Accepted 15 December 2015

Academic Editor: Sofia Lionaki

Copyright (C) 2015 Sophie Van Haare Heijmeijer et al. This is an open access article distributed under the Creative Commons Attribution License, which permits unrestricted use, distribution, and reproduction in any medium, provided the original work is properly cited.

Infective endocarditis (IE) and small-vessel vasculitis may have similar clinical features, including glomerulonephritis. Furthermore the association between IE and ANCA positivity is well documented, making differential diagnosis between IE- and ANCAassociated vasculitis particularly difficult, especially in case of culture-negative IE. We report on one patient with glomerulonephritis secondary to culture-negative IE caused by Bartonella henselae which illustrates this diagnostic difficulty.

\section{Introduction}

Infective endocarditis (IE) and small-vessel vasculitis may have a similar clinical presentation. Cardiac involvement has been documented in patients with Wegener granulomatosis [1]. Conversely, patients with IE often have renal complications, including glomerulonephritis. Moreover the association between IE and ANCA positivity is well documented, making differential diagnosis between IE- and ANCA-associated vasculitis particularly difficult, especially in case of culture-negative IE. We report on one patient with glomerulonephritis secondary to culture-negative IE caused by Bartonella henselae which highlights this diagnostic difficulty.

\section{Case}

A 67-year-old man presented with asthenia and weight loss (12 kg over 5 months). His medical history included hypertension, bicuspid aortic valve, and thoracic aortic aneurysm repair. He owned four pet dogs and one cat. Medications included amlodipine and acetylsalicylic acid. There was no history of recent dental procedure or use of intravenous drugs. He did not report fever, chills, night sweats, hematuria, or oliguria. On admission he was afebrile and normotensive. Physical examination revealed palpable purpura on his legs without edema.

Three months prior to admission, serum creatinine and CRP levels were $1.6 \mathrm{mg} / \mathrm{dL}$ [eGFR $43 \mathrm{~mL} / \mathrm{min} / 1.73 \mathrm{~m}^{2}$ by the 4-variable MDRD (Modification of Diet in Renal Disease Study equation)] and $2.1 \mathrm{mg} / \mathrm{dL}$, respectively. On admission laboratory data revealed serum creatinine $3.5 \mathrm{mg} / \mathrm{dL}$ (eGFR $18 \mathrm{~mL} / \mathrm{min} / 1.73 \mathrm{~m}^{2}$ ), serum urea $88 \mathrm{mg} / \mathrm{dL}$, hemoglobin $8.8 \mathrm{~g} /$ $\mathrm{dL}$, normal platelet count, and moderate inflammatory syndrome (CRP $2.3 \mathrm{mg} / \mathrm{dL}$ ) with polyclonal hypergammaglobulinemia. Urinalysis showed hematuria (1370 red blood cells $/ \mu \mathrm{L}$ ) with red blood cell casts and moderate proteinuria (protein/creatinine ratio $0.72 \mathrm{~g} / \mathrm{g} ; 830 \mathrm{mg} / 24 \mathrm{~h}$ ). Further laboratory investigations revealed both positive myeloperoxidase antineutrophil cytoplasmic (MPO-ANCA) $(89 \mathrm{RU} / \mathrm{mL}$, nor$\mathrm{mal}<20)$ and proteinase 3 antibodies (PR3-ANCA) (41 RU/ $\mathrm{mL}$, normal <20); low complement levels of C3 $(80 \mathrm{mg} / \mathrm{dL}$; normal $90-180 \mathrm{mg} / \mathrm{dL}$ ); normal C4 levels; and increased rheumatoid factor $(189 \mathrm{IU} / \mathrm{mL}$; normal <40). Anti-nuclear 


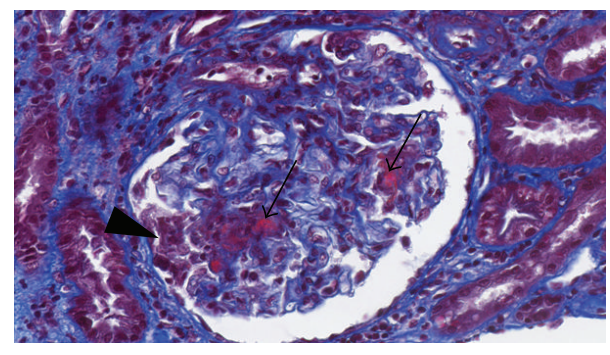

Figure 1: Kidney biopsy findings (Masson's trichrome, 46x): glomeruli showing foci of necrosis (arrows) with a recent cellular crescent (arrowhead).

and anti-cardiolipin antibodies were negative. There was no cryoglobulinemia. Serologic tests for hepatitis B, C and HIV were negative. Multiple blood cultures were negative.

Renal biopsy showed segmental necrosis involving 1 out of 16 nonsclerotic glomeruli, as well as one cellular crescent and one fibrous crescent (Figure 1). Two glomeruli were globally sclerosed. Foci of acute interstitial infiltrate and acute tubular necrosis were associated. Mild significant interstitial fibrosis was observed. Immunofluorescence showed moderate granular parietal staining for IgM, C3, and Clq (Figure 2), which suggested infection-related glomerulonephritis. However transesophageal echocardiography did not show any signs of endocarditis. Imaging by positron-emission tomography only showed mild splenomegaly.

Because of this rapidly progressive renal failure secondary to necrotizing and crescentic glomerulonephritis without any evidence of infective endocarditis, treatment with cyclophosphamide (500 mg) and intravenous pulses of methylprednisolone was started, followed by oral corticotherapy $(1 \mathrm{mg} / \mathrm{kg} /$ day $)$. The patient felt rapidly better and renal function improved.

However, ten days later, creatinine level acutely peaked at $9.2 \mathrm{mg} / \mathrm{dL}$ and hemodialysis was started. Two weeks later the patient presented with thoracic pain. Coronary angiography showed a double coronary vessel disease. Repeated transesophageal echocardiography showed severe aortic and moderate mitral insufficiency with possible perforation. Broad-spectrum antibiotics were started and the patient underwent mitral valve repair, aortic valve replacement, and triple coronary artery bypass. He had an uneventful postoperative course. Hemodialysis therapy was discontinued 13 days later. Histopathological examination of the excised valve was consistent with bacterial endocarditis, but cultures of the valve remained negative. Serological test results for atypical pathogens turned negative except for Bartonella henselae (IgM: 1/100; IgG: 1/1280). Bartonella henselae DNA was detected by polymerase chain reaction (PCR) on the resected valve. The patient was administered doxycycline and gentamycin for two weeks. He was discharged home and continued treatment by doxycycline and rifampicin for four more weeks. Methylprednisolone doses were slowly tapered. Three months later, creatinine level was $1.5 \mathrm{mg} / \mathrm{dL}$, and ANCA were negative.

\section{Discussion}

Renal disease in the setting of IE is a well-known extracardiac complication of IE, affecting as many as $40-50 \%$ of patients with IE [2]. Kidney lesions include abscess from septic emboli, immune complex-mediated glomerulonephritis, ANCA-associated glomerulonephritis, and renal toxicity secondary to antibiotics.

Among the causes of infection-related glomerulonephritis in adults, 6-20\% are related to endocarditis [3] and glomerulonephritis is considered as a minor Duke criterion for the diagnosis of IE [4]. In a recent large biopsy-based clinicopathologic series on IE-related glomerulonephritis ( $n$ $=49$ ), acute kidney injury was the most common presenting condition (79\%) with hematuria present in almost all cases. However, typical nephritic and nephrotic syndromes were relatively uncommon (9 and 6\%, resp.) [5]. Hypocomplementemia (low C3 and/or C4 levels) was found in 56\% of patients tested and ANCA antibodies in as many as $28 \%$, with mostly anti-PR3 specificity, although anti-MPO and dual-positive anti-PR3 and anti-MPO were also seen. As in previous reports (summarized in [3]), the most frequent infectious organism identified on blood cultures was Staphylococcus aureus (53\%), followed by Streptococcus species (23\%) [5]. Importantly, culture-negative endocarditis accounts for almost $10 \%$ of the patients with IE-related glomerulonephritis $[5,6]$.

The spectrum of glomerular histopathologic lesions of IE-related glomerulonephritis is variable. Diffuse necrotizing crescentic GN is the most common pattern (53\%), followed by diffuse proliferative glomerulonephritis (37\%) and mild mesangial hypercellularity without endocapillary proliferation or crescents (10\%) [5]. The immunofluorescence patterns include classic postinfectious type (with prominent IgG and C3 deposition and subepithelial humps on electron microscopy), although IgM- or IgA-dominant staining (particularly with Staphylococcus aureus) has also been reported with variable frequency $[3,5,7]$. Interestingly, the association between IE and pauci-immune glomerulonephritis has been documented in as many as $44 \%$ of cases [5].

Our patient has experienced blood culture negative endocarditis, which represents $2.5-31 \%$ of all cases of IE [8]. The most often, previous antibiotic therapy is the reason for negative blood cultures. The namely "true" blood culture-negative IE is caused by intracellular bacteria that cannot be routinely cultured in blood with currently available techniques. In a large reference laboratory series, Bartonella sp. was the second most common aetiology in Europe (28\%), after Coxiella burnetii [8]. B. quintana has been associated with body-louse infested alcoholic homeless persons, whereas $B$. henselae has been associated with patients with exposure to cats or their fleas, and previous structural valvulopathy, with most cases occurring in native aortic valves [8].

Diagnostic tests for Bartonella IE include cultures and serologic and DNA amplification techniques. PCR from valvular tissue has been demonstrated to have a higher sensitivity than blood and valvular culture (92\% versus 20 and $31 \%$, resp.), even in patients receiving antibiotics [8]. In a recent large series of Bartonella IE, serum Western 


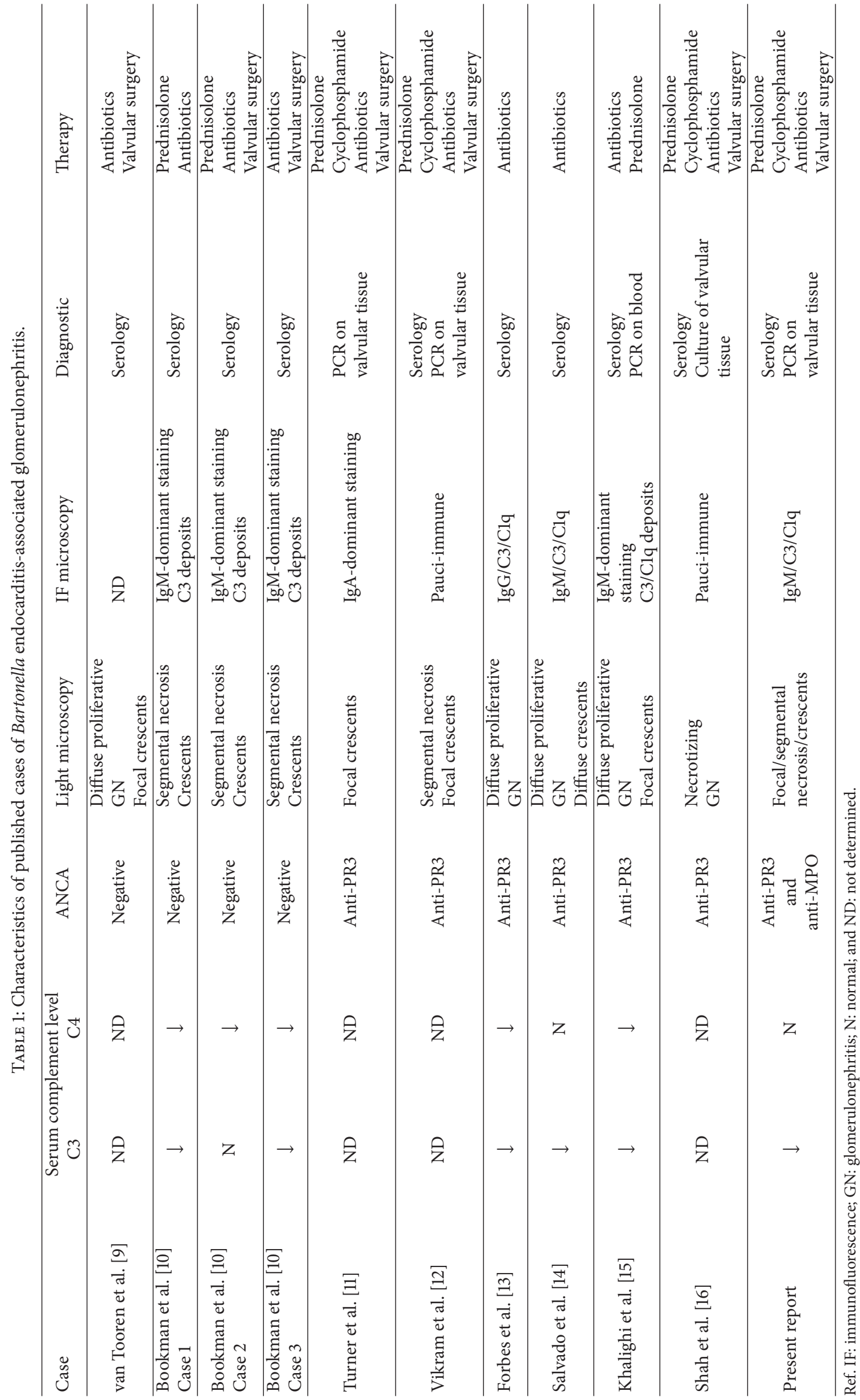



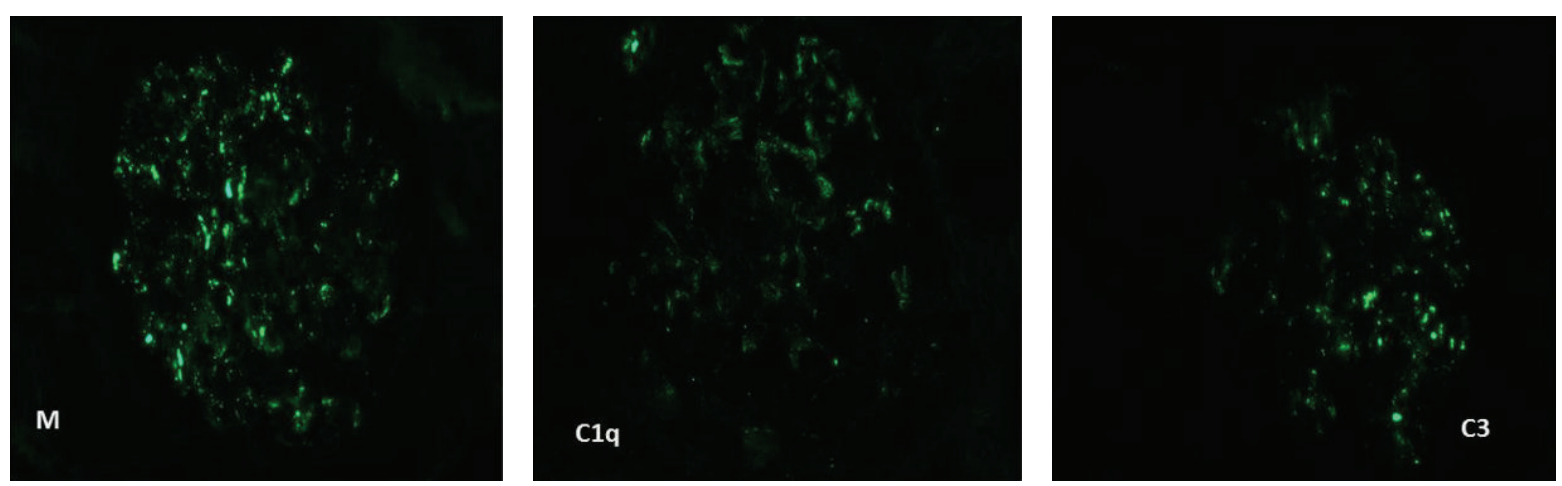

FIGURE 2: Immunofluorescence shows subepithelial granular staining for IgM and C3 and parietal granular staining for Clq.

blotting (WB), PCR, and 16S rRNA gene amplification in valvular tissue exhibited sensitivities of $100 \%, 92 \%$, and $60 \%$, respectively [17]. Although PCR on total blood and serum may enable the diagnosis before cardiac surgery, its sensitivity is low (33\% and 36\%, resp.) [17]. Bartonella species IgG titer $>1: 800$ using an immunofluorescence assay has a predictive value of $95 \%$ to detect Bartonella IE, but a titer $<800$ does not exclude the diagnosis $[17,18]$. Therefore, any patient with IgG titer $<800$ and a medical history evocative of IE should be tested by WB and PCR following cardiac valve removal. Currently, it is considered that a positive PCR from a cardiac valve or blood specimen, an IgG titer of $>800$, and a positive WB assay should be major criteria for Bartonella IE [17].

Glomerular disease in the setting of Bartonella IE seems to be a rare complication, but its true incidence is unknown. To the best of our knowledge, there have been only 10 cases published to date (Table 1); all of them are caused by $B$. henselae. Hypocomplementemia was documented in the 6 patients in whom complement levels were tested. ANCA were positive in 7/11 cases, with anti-PR3 specificity in 6 . Of note, dual anti-PR3 and anti-MPO specificity was documented in only one case (this present case). The predominant light microscopy pattern of injury was segmental necrotizing crescentic glomerulonephritis. On immunofluorescence, the predominant immunoglobulin was $\operatorname{IgM}$, with strong C3 deposits, although in two cases there was a dominant staining for IgA [11] and IgG [14]. Only two cases showed a pauciimmune pattern. Most cases required valvular surgery. Interestingly, in 7/11 cases, immunosuppressive agents were first administered for a suspected vasculitis and withdrawn when the diagnosis of endocarditis was made.

This case highlights the difficulty to make the differential diagnosis between occult infection with secondary glomerular involvement and ANCA-associated vasculitis. Distinguishing these two conditions is crucial, particularly when immunosuppressive treatment is considered. Although delayed, the diagnosis of culture-negative IE was finally suggested by a proliferative immune complex-mediated glomerulonephritis in a patient with a structural cardiac valve anomaly. The association of rapidly progressive renal failure secondary to necrotizing and crescentic glomerulonephritis and palpable purpura on the legs without any evidence of IE has initially supported the diagnosis of ANCAassociated vasculitis. However, even in case of negative blood cultures, the concomitant presence of hypocomplementemia and positive ANCA antibodies is strongly suggestive of immune complex-mediated disease. Moreover the dualpositive ANCA specificity anti-PR3 and anti-MPO is very unusual and should always trigger the screening for secondary causes of ANCA positivity, that is, occult infection, including IE due to atypical pathogens [5], or levamisolecontaminated cocaine use [19].

\section{Conflict of Interests}

The authors declare that there is no conflict of interests regarding the publication of this paper.

\section{Authors' Contribution}

Sophie Van Haare Heijmeijer and Dunja Wilmes have contributed equally to this paper.

\section{References}

[1] G. S. Hoffman, G. S. Kerr, R. Y. Leavitt et al., "Wegener granulomatosis: an analysis of 158 patients," Annals of Internal Medicine, vol. 116, no. 6, pp. 488-498, 1992.

[2] D. J. Sexton and D. Spelman, "Current best practices and guidelines. Assessment and management of complications in infective endocarditis," Cardiology Clinics, vol. 21, no. 2, pp. 273282, 2003.

[3] S. H. Nasr, J. Radhakrishnan, and V. D. D’Agati, "Bacterial infection-related glomerulonephritis in adults," Kidney International, vol. 83, no. 5, pp. 792-803, 2013.

[4] J. S. Li, D. J. Sexton, N. Mick et al., "Proposed modifications to the Duke criteria for the diagnosis of infective endocarditis," Clinical Infectious Diseases, vol. 30, no. 4, pp. 633-638, 2000.

[5] C. L. Boils, S. H. Nasr, P. D. Walker, W. G. Couser, and C. P. Larsen, "Update on endocarditis-associated glomerulonephritis," Kidney International, vol. 87, no. 6, pp. 1241-1249, 2015.

[6] P.-E. Fournier, F. Thuny, H. Richet et al., "Comprehensive diagnostic strategy for blood culture-negative endocarditis: a 
prospective study of 819 new cases," Clinical Infectious Diseases, vol. 51, no. 2, pp. 131-140, 2010.

[7] A. Majumdar, S. Chowdhary, M. A. S. Ferreira et al., "Renal pathological findings in infective endocarditis," Nephrology Dialysis Transplantation, vol. 15, no. 11, pp. 1782-1787, 2000.

[8] P. Houpikian and D. Raoult, "Blood culture-negative endocarditis in a reference center: etiologic diagnosis of 348 cases," Medicine, vol. 84, no. 3, pp. 162-173, 2005.

[9] R. M. van Tooren, R. van Leusen, and F. H. Bosch, "Culture negative endocarditis combined with glomerulonephritis caused by Bartonella species in two immunocompetent adults," Netherlands Journal of Medicine, vol. 59, no. 5, pp. 218-224, 2001.

[10] I. Bookman, J. W. Scholey, S. V. Jassal, G. Lajoie, and A. M. Herzenberg, "Necrotizing glomerulonephritis caused by Bartonella henselae endocarditis," American Journal of Kidney Diseases, vol. 43, no. 2, pp. e25-e30, 2004.

[11] J. W. Turner, B. C. Pien, S. A. Ardoin et al., "A man with chest pain and glomerulonephritis," The Lancet, vol. 365, no. 9476, article 2062, 2005.

[12] H. R. Vikram, A. K. Bacani, P. A. DeValeria, S. A. Cunningham, and F. R. Cockerill III, "Bivalvular Bartonella henselae prosthetic valve endocarditis," Journal of Clinical Microbiology, vol. 45, no. 12, pp. 4081-4084, 2007.

[13] S. H. Forbes, S. C. Robert, J. E. Martin, and R. Rajakariar, "Acute kidney injury with hematuria, a positive ANCA test, and low levels of complement," American Journal of Kidney Diseases, vol. 59, no. 1, pp. A28-A31, 2012.

[14] C. Salvado, A. Mekinian, P. Rouvier, P. Poignard, I. Pham, and O. Fain, "Rapidly progressive crescentic glomerulonephritis and aneurism with antineutrophil cytoplasmic antibody: Bartonella henselae endocarditis," Presse Medicale, vol. 42, no. 6, pp. 10601061, 2013.

[15] M. A. Khalighi, S. Nguyen, J. A. Wiedeman, and M. F. Palma Diaz, "Bartonella endocarditis-associated glomerulonephritis: a case report and review of the literature," American Journal of Kidney Diseases, vol. 63, no. 6, pp. 1060-1065, 2014.

[16] S. H. Shah, C. Grahame-Clarke, and C. N. Ross, "Touch not the cat bot a glove: ANCA-positive pauci-immune necrotizing glomerulonephritis secondary to Bartonella henselae," Clinical Kidney Journal, vol. 7, no. 2, pp. 179-181, 2014.

[17] S. Edouard, C. Nabet, H. Lepidi, P. E. Fournier, and D. Raoult, "Bartonella, a common cause of endocarditis: a report on 106 cases and review," Journal of Clinical Microbiology, vol. 53, no. 3, pp. 824-829, 2015.

[18] P.-E. Fournier, J.-L. Mainardi, and D. Raoult, "Value of microimmunofluorescence for diagnosis and follow-up of Bartonella endocarditis," Clinical and Diagnostic Laboratory Immunology, vol. 9, no. 4, pp. 795-801, 2002.

[19] A. Q. Carlson, D. S. Tuot, K.-Y. Jen et al., "Pauci-immune glomerulonephritis in individuals with disease associated with levamisole-adulterated cocaine: a series of 4 cases," Medicine, vol. 93, no. 17, pp. 290-297, 2014. 


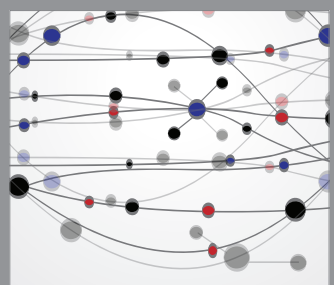

The Scientific World Journal
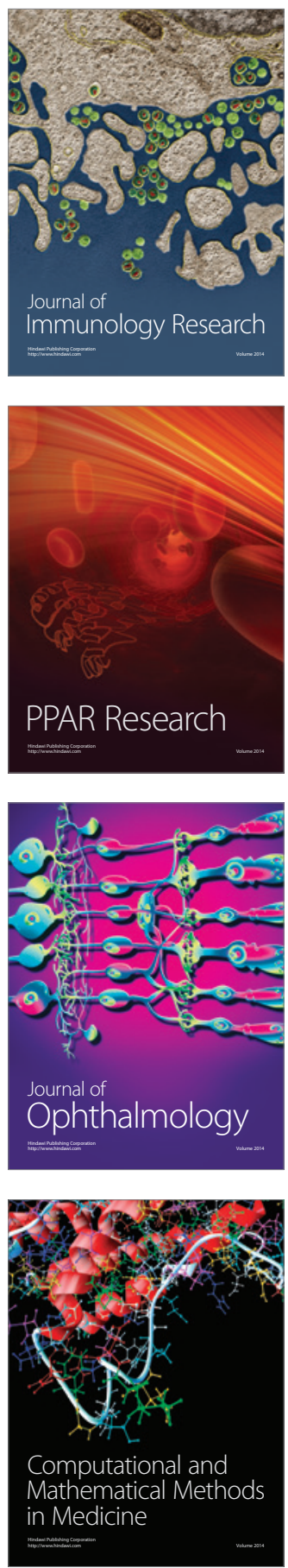

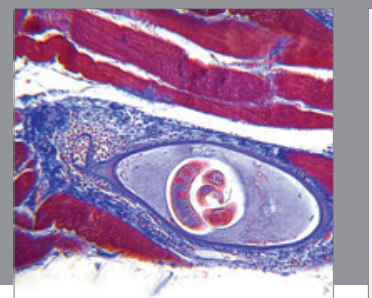

Gastroenterology

Research and Practice
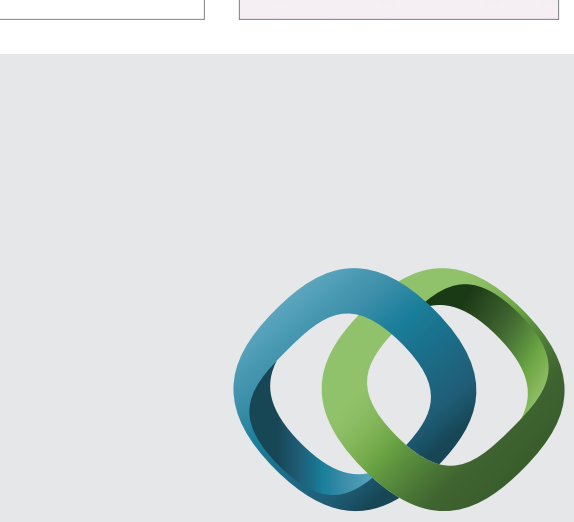

\section{Hindawi}

Submit your manuscripts at

http://www.hindawi.com
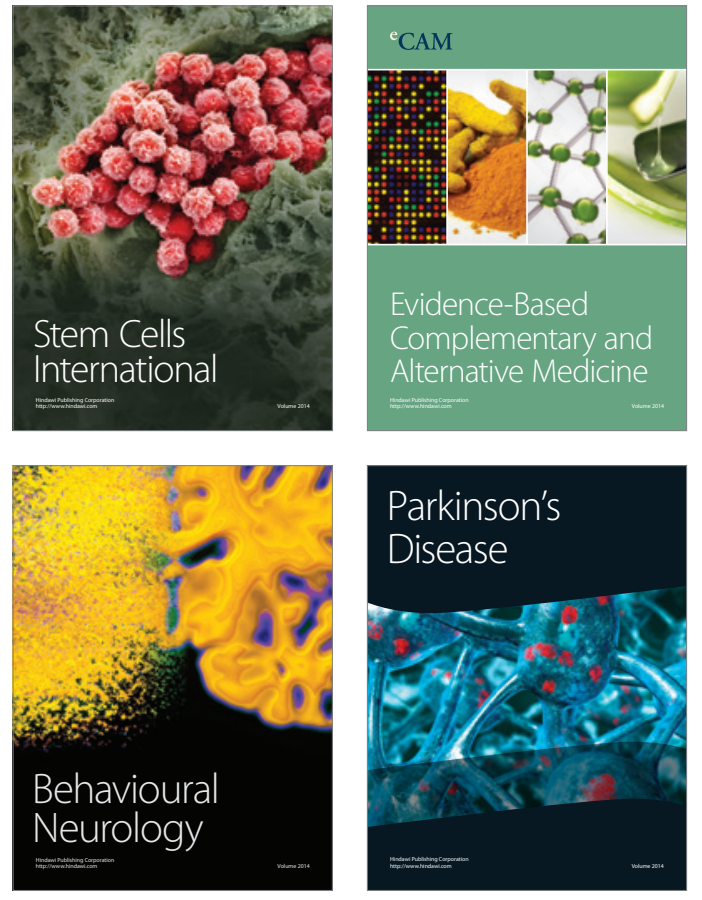
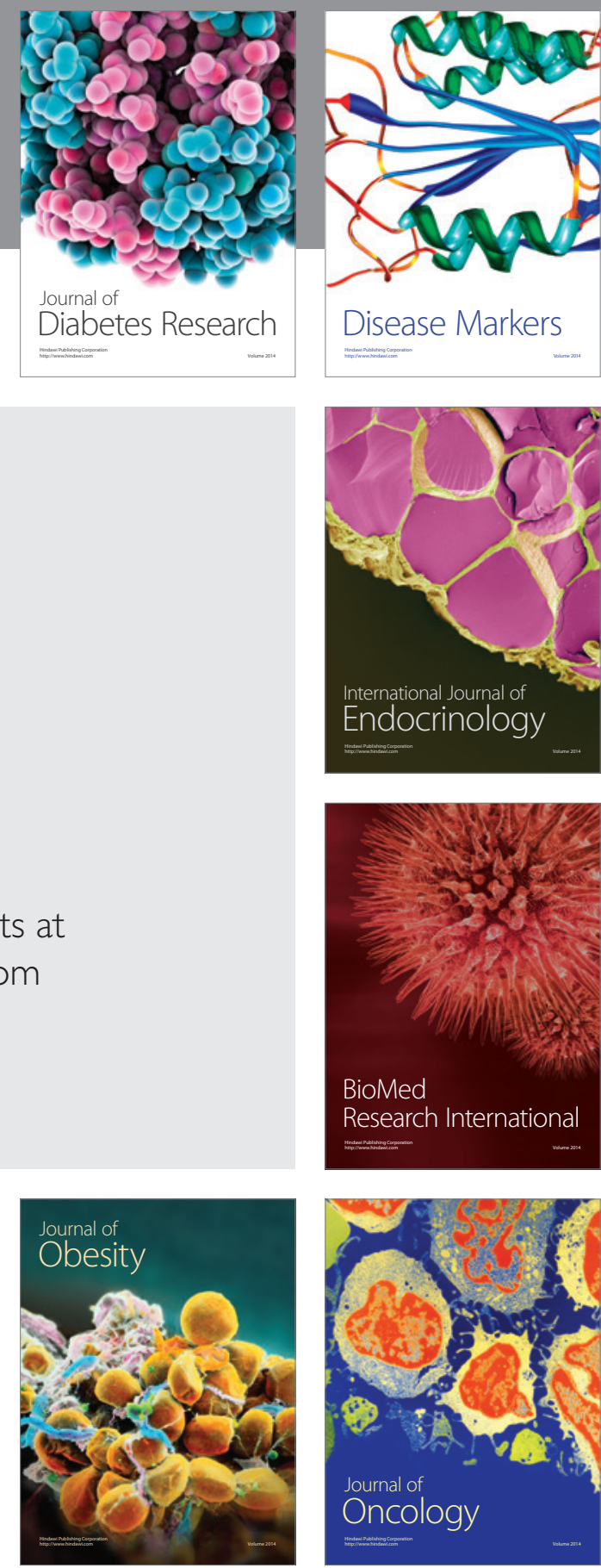

Disease Markers
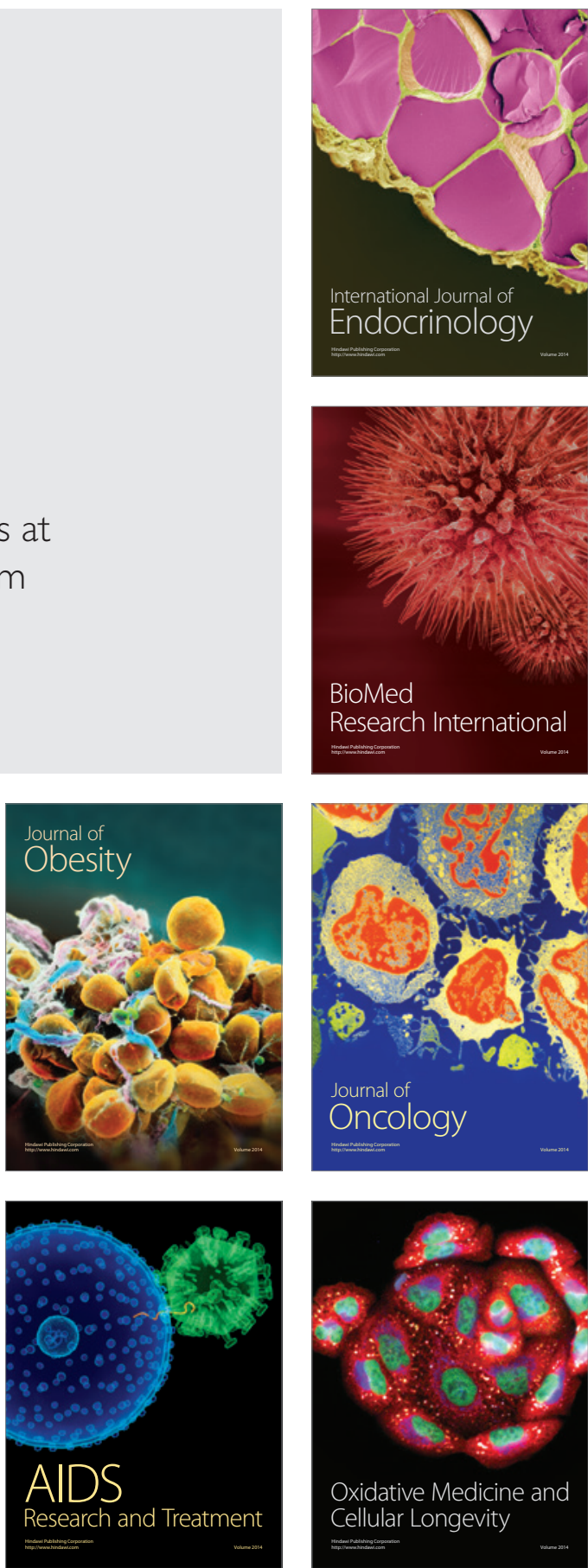